\title{
Synthesis and Surface Derivatization of Processible Co Nanoparticles
}

\author{
Jin-Kyu Lee and Sung Moon Choi \\ School of Chemistry and Molecular Engineering, Seoul National Limersin, Seoul 151-747. Korea \\ Received.August 22, 2002
}

\begin{abstract}
Co nanoparticles were prepared by the reverse micelle technique $\left(\mathrm{NaBH}_{4}\right.$ reduction of cobalt chloride in a reversed micelle solution of didodecyldimethy lammoniumbromide (DDAB)/toluene). The size and the shape of Co nanoparticles could be easily controlled by changing the water contents and micelle concentrations. and the solubility of Co nanoparticles was systematically tuned by choosing appropriate surface capping organic ligand molecules. Furthermore, a novel nanofabrication process was clearly demonstrated. which generated oxide over-coated Co nanorods from Co nanoparticles in organic solution by slow oxidation with an external magnetic field.
\end{abstract}

Key Words : Co nanoparticle, Nanorod, Surface modification, Reverse nucelle

\section{Introduction}

Recently. as an essential component of nanotechnology in the $21^{\text {st }}$ century. materials consisting of particles with dianteter less than $100 \mathrm{~nm}$ have attracted a great deal of attention. Owing to their nanometer scale size, these particles lead to drantatic changes in physical and chemical properties (i.e.. novel properties that differ considerably from those of molecules as well as bulk materials). which could be utilized in many important applications. ${ }^{1-5}$ Especially. magnetic nanoparticles have been used as the active component of ferrofluids, magnetic recording devices. electronic components, solar energy transformers, and chemical catalysts. Furthermore. superparamagnetic nanoparticles have also found applications in medicine. for example. in drug delivery and restriction of blood flow to a selected part of the body. ${ }^{6}$ For high-density magnetic recording applications, new magnetic materials require particles of small size, narrow size distribution, as well as control of size and shape. The fabrication methods used to organize magnetic nanoparticles are also becoming increasingly important

Numerous physical and chemical methods have been developed for the preparation of magnetic nanoparticles. ${ }^{7-12}$ Bloomfield et $a l^{13.14}$ and De Heer et $a l^{15}$ employed laser ablation methods and prepared magnetic nanoparticles ( $\mathrm{Co}$. $\mathrm{Ni} . \mathrm{Fe}$ ) of $40-500$ atoms. However these nanoparticles were not stable in air due to oxidation and aggregation because their surfaces were not passivated by other organic or inorganic molecules (bare nanoparticles). One popular synthetic method for the preparation of nanoparticles is a socalled reverse micelle technique that can prevent aggregation of the nanoparticles and possible oxidation from the air. Furthermore. surface modification (derivatization or capping) of the nanoparticles in the solution is a useful means of dispersing them in a variety of media and of mediating the growth process. ${ }^{4.16}$ Although some studies on the surface modification of nanoparticles have recently been reported ${ }^{17-19}$

*To whom all correspondence should be addressed: Phone: $+82-$ 2-879-2923, Fax: +82-2-882-1080, E-mail: jinklee $t$ snu. ac kr the development of organic- or water-soluble cobalt nanoparticles by adopting the reverse micellar teclunique has not yet been covered. In addition. magnetic dipole-dipole attraction between particles enlances the difficulties experienced in the production of ferrofluids in comparison to nonmagnetic namoparticles. The particle agglomeration is one of the critical obstacles to be overcome in producing stable magnetic fluids. In these respects, surface modification of magnetic nanoparticles with various organic materials could provide a breakthrough for the production of stable magnetic fluids, since the coating (or capping) materials might act as a stabilizer and are believed to prevent agglomeration of magnetic nanoparticles. In addition, it is believed that the solvent compatibility of magnetic nanoparticles can be easily tailored by deliberately choosing the capping materials and their usability can be greatly extended for practical applications (e.g.. catalysis. biological cell separation, raw material recovery. drug delivery, anti-corrosion protection, etc.) ${ }^{3 j, 21}$

We have adapted a mild and simple 'reverse micelle technique" to prepare highly processible cobalt nanoparticles. "2 The well-defined size and shape of the product could be controlled by the amount of water contents and micelle concentrations. Furthermore. cobalt nanoparticles could be stabilized by passivating the nanoparticle surfaces with various organic capping ligands such as thiophenol (TP), dodecanethiol (DT), pentafluorothiophenol (PFTP). and 11-mercaptonudecanoic acid (11-MUA). These surfacederivatized Co nanoparticles exhibit versatile solubility in various solvents. which could make them useful for many practical applications.

\section{Experimental Section}

Materials: The surfactant didodecyldimethỵlammoniumbromide (DDAB. 98\%). was purchased from T.C.I. and used as received. Cobalt chloride hexahydrate $\left(\mathrm{CoCl}_{2} 6 \mathrm{H}_{2} \mathrm{O}\right.$. $98 \%$ ) and sodium borohydride $\left(\mathrm{NaBH}_{4}\right)$ were purchased from Duck-San Chem. Co. Korea. Capping materials were obtained from the following sources and used without 
further purification: thiophenol ( $98 \%$. T.C.I.). dodecanethiol (97\%. Aldrich). 4-aminothiophenol $(97 \%$. Aldrich), and pentafluorothiophenol $(95 \%$. Aldrich). All the solvents for the synthesis were purified to remove moisture and oxygen by the known literature methods. 3

\section{Preparation of Co nanoparticles}

Bare Co nanoparticle: All the synthesis steps were carried out in an $\mathrm{N}_{2}$ environment to avoid possible oxygen contamination during the reaction. Dried toluene and deionized water were bubbled with $\mathrm{N}_{2}$ gas for $2 \mathrm{~h}$ before the experiment to remove oxygen. A typical preparation procedure for making Co nanoparticles is described in the following: In a $25 \mathrm{~mL}$ two neck flask. DDAB (1.5 g. 3.0 mmol) was dissolved in $10 \mathrm{~mL}$ of dried-toluene to form a 0.3 $\mathrm{M}$ reverse micelle solution. (For preparation of $0.4 \mathrm{M}$ reverse micelle solution. $8 \mathrm{~mL}$ instead of $10 \mathrm{~mL}$ of driedtoluene was used.) This reverse micelle solution was sonicated to make a clear solution and $\mathrm{CoCl}_{2} \cdot 6 \mathrm{H}_{2} \mathrm{O}(25.00 \mathrm{mg} .0 .1$ mmol) and an appropriate amount of water $(20$ or $40 \mu \mathrm{L}$ ) was then added. The mixed solution was sonicated until the entire solid disappeared and a clear blue solution was obtained. $\mathrm{NaBH}_{4}(113 \mathrm{mg}$ ) was dissolved in $3 \mathrm{~mL}$ of deionized water to prepare $1 \mathrm{M}$ solution. and $300 \mu \mathrm{L}$ of $1 \mathrm{M} \mathrm{NaBH}_{4}$ solution was slowly added to the reverse micelle solution using a microsyringe while the solution was vigorously stirring. A stable black colloid solution appeared after I min.

Organic-soluble Co nanoparticles: In the very last step of the general procedure described above. inmediately after Co nanoparticles were produced. a large amount $(150 \mu \mathrm{L})$ of thiophenol (TP) was added. The reaction mixture was stirred for one day to ensure a complete reaction, and the resulting solution turned optically clear. The surface-modified cobalt nanoparticles were then precipitated fron the solution by adding an excess amount of absolute methanol. Dodecanethiol (DT) and pentafluorothiophenol (PFTP) capped Co nanoparticles were similarly prepared by using dodecanethiol and pentafluorothiophenol as capping reagents except for the use of absolute ethanol and acetonitrile instead of methanol to precipitate for DT-capped and PFTP-capped Co nanoparticles. respectively: These suspensions were diluted with acetone and the solid products were isolated from the solution by the magnetic decantation technique. This decantation procedure was repeated 2-3 times to remove excess surfactants and capping materials. Dark gray solid products were obtained after drying under $\mathrm{N}_{3}$, and these surface-modified Co nanoparticles were then easily redispersed in various solvents. This surface passivation treatment with various organic materials strongly improved the stability of Co nanoparticles from the aggregation. and the nanocluster solution could remain clear without forming insoluble precipitates for more than a few months.

Water-soluble Co nanoparticles: An excess amount of 11-mercaptonudecanoic acid (MUA) was added to thiophenol-capped cobalt nanoparticles. The mixture was stirred and heated at $100^{\circ} \mathrm{C}$ under $\mathrm{N}_{2}$ conditions for $24 \mathrm{~h}$ to cause MUA to replace the thiophenol on the surface of $\mathrm{Co}$ nanoparticles. Absolute methanol was added to the mixture solution to remove the excess amount of MUA. The reddish pink color Co nanoparticle was precipitated slowly and was collected by centrifugation. After the precipitates were dissolved in THF, $6 \mathrm{~N} \mathrm{NaOH}$ solution was slowly added to neutralize the terminal acid groups of MUA and form ionic salts. The pink precipitates were isolated to show high solubility in water producing clear aqueous Co nanoparticle solution.

Instruments: Transmission electron microscopy (TEM) images and electron diffraction pattenns were obtained on a JEOL $100 \mathrm{CX} 2$ operating at $200 \mathrm{kV}$. A powder of thiophenol capped Co nanoparticles $(1.0 \mathrm{mg}$ ) was dissolved in $2 \mathrm{~mL}$ of DMSO. One drop of this solution was diluted with $2 \mathrm{~mL}$ DMSO. A drop of the dilute solution was placed on a carbon coated copper grid and the solvent was evaporated under a nitrogen atmosphere.

Infrared spectra were recorded on the Bomem DA8 spectrometer equipped with a Michelson-type interferometer with $0.03 \mathrm{~cm}^{-1}$ resolution in transmission mode accumulating 32 scans at room temperature. Mixtures of poivder samples and $\mathrm{KBr}$ were finely ground, pressed into diskshaped pellets, and used for IR measurements.

$\mathrm{X}$-ray photoelectron spectra were measured using $\mathrm{PHI}$ Model 5700 Multi Teclunique Systems with an Al X-ray source $(\mathrm{Al} \mathrm{K} / x=1486.6 \mathrm{eV})$.

\section{Results and Discussion}

Cobalt nanoparticles were synthesized with different water contents and reverse micelle concentrations. and their size and shape were studied by TEM. Figure l(a) shows TEM images of a monolayer assembly of spherical cobalt nanoparticles prepared with a water content of $20 \mu \mathrm{L}$. The nanoparticles in the size range of $8-10 \mathrm{~cm}$ are homogeneously dispersed without any noticeable aggregates. It has been reported that in this size range, ferromagnetism of magnetic nanoparticles transforms into superparamagnetism. ${ }^{24}$ The Co nanoparticles with a narrow size distribution could easily form a monolayer structure with a hexagonal closest packed network on the TEM grid because these nanoparticles are likely to be superparamagnetic and the interparticle magnetic interaction is negligible. Figure l(b) shows a TEM image of spherical cobalt nanoparticles with an average size of $50 \mathrm{~nm}$. prepared in the condition with 40 $\mu \mathrm{L}$ of water. As expected. the size of cobalt nanoparticles can be varied in a certain range by changing the amount of water content in the reverse micelle. A reverse micelle is a nanometer size cage fomed by dissolving surfactant molecules in an apolar organic solvent (i.e., the hydrophilic ends of the surfactant molecules spontaneously gather together to form a cavity while hydrophobic ends are sticking out to the solvent.) Therefore the size of this cage can be varied by the amount of water contained inside the cage.

When the concentration of reverse micelle was increased from $0.3 \mathrm{M}$ to $0.4 \mathrm{M}$. needle shape cobalt nanoparticles were obtained as shown in Figure 2. Wang et al. have reported the 


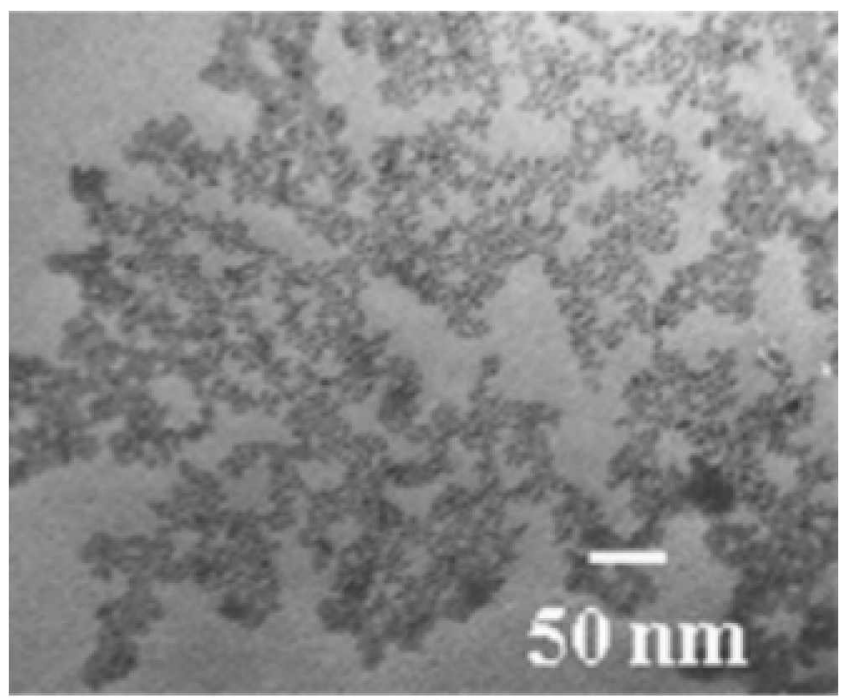

(a)

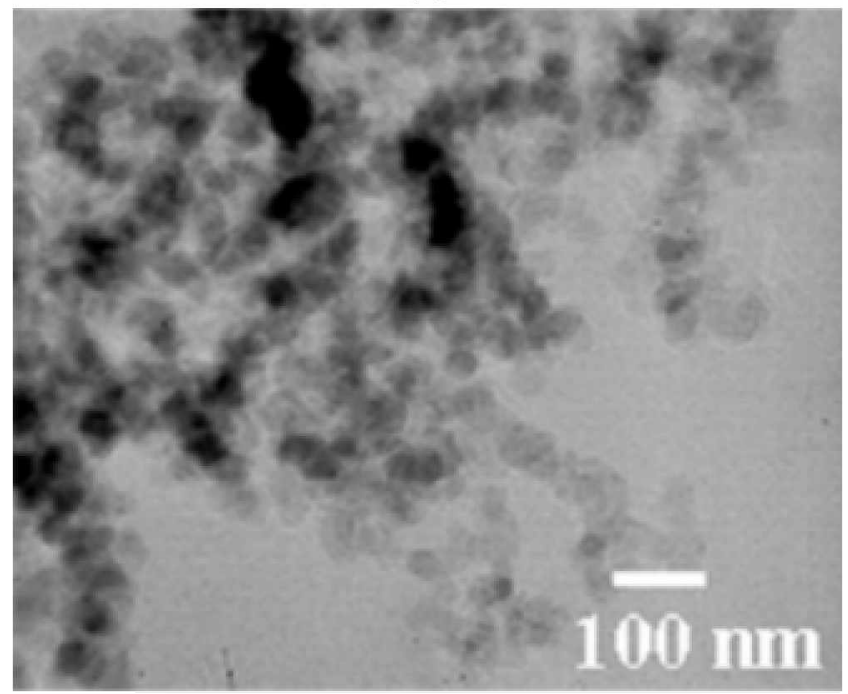

(b)

Figure 1. TEM images of the cobalt nanoparticles synthesized from $0.3 \mathrm{M}$ reverse nincelle concentration (a) with water content of $20 \mu \mathrm{L}$ (b) with water content of $40 \mu \mathrm{L}$.

fornation of self-assembling whisker-shaped cobalt nanoparticles by applying an extemal magnetic field during the drying process. ${ }^{-4}$ Recently some detailed studies on the transition of micelle shape in the micellar solution have been reported. ${ }^{-5-29}$ The Alivisatos group and Pileni group have also reported that the shapes and sizes of metal nanoparticles could be controlled by using binary surfactant sy'stem or varying the surfactant concentration. ${ }^{31 \cdot 32}$ Under the condition with a fixed concentration of $\mathrm{Co}^{2+}$ as reported in the literature ${ }^{33}$ the aspect ratio of Co nanoparticles increases with increasing amount of surfactant (i.e. DDAB) in reverse micelle solution.

Bare Co nanoparticles were not very stable when they were isolated from the preparation solution containing a large amount of surfactant (DDAB), forming aggregates that were totally insoluble in any solvents. In order to enhance the stability of Co nanoparticles. the reactive surface was

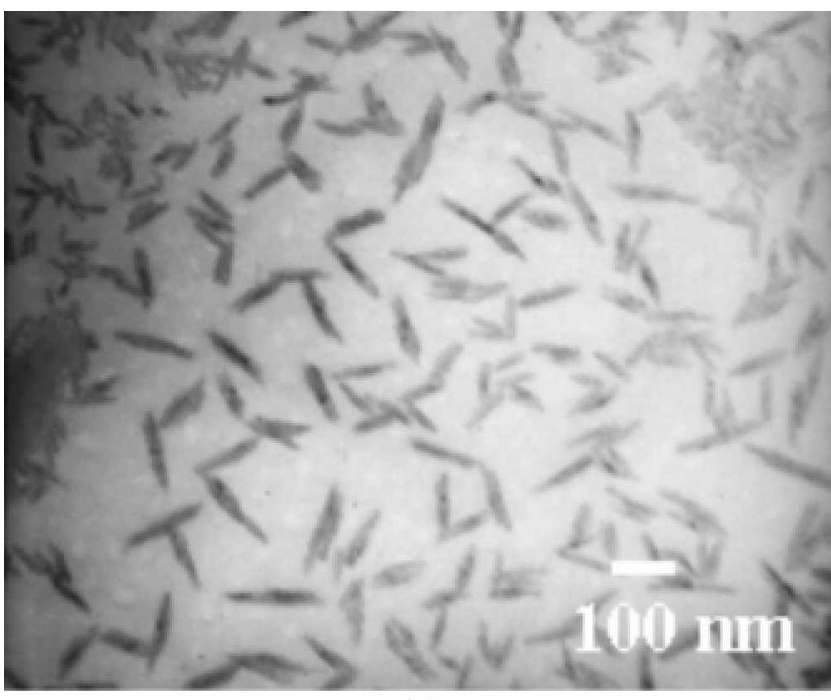

(a)

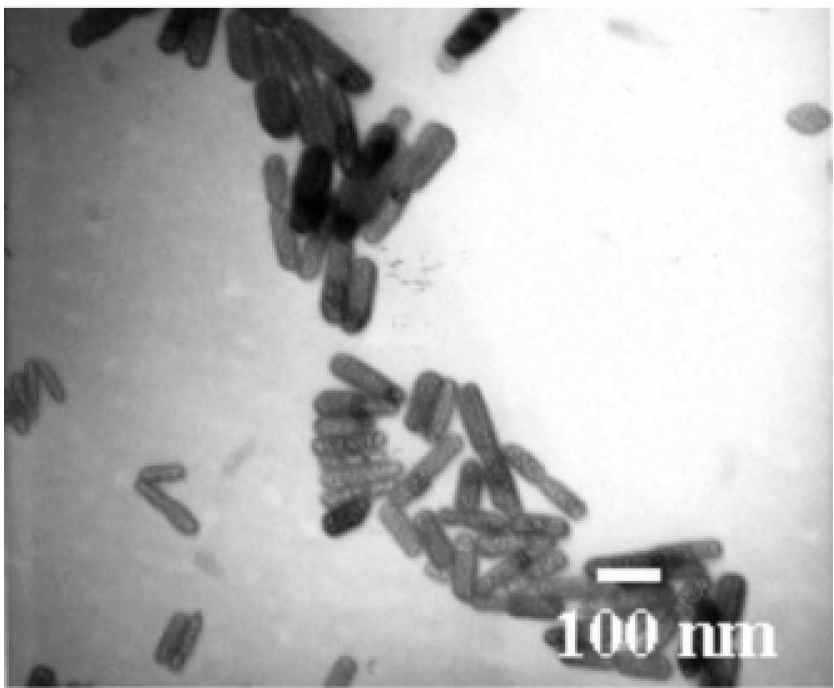

(b)

Figure 2. TEM images of the cobalt nanoparticles synthesized from $0.4 \mathrm{M}$ reversemicelle concentration with different water contents; (a) $20 \mu \mathrm{L}$ and (b) $40 \mu \mathrm{L}$.

passivated (capped) with various organic compounds. These organic-capped cobalt nanoparticles showed extreme stability enhancement compared to bare nanoparticles and they could be easily redispersed in various organic solvents (Table l). Thiophenol(TP)-capped Co nanoparticles are soluble in relatively polar solvents such as pyridine. MIBK. and DMSO, but insoluble in relatively nonpolar solvents such as

Table 1. Solubility of various organic capped cobalt nanoparticles

\begin{tabular}{lc}
\hline Variouls Capped Co Nanoparticles & Soluble solvents \\
\hline Thiophenol capped Co nanoparticles & pyridine, MBK, \\
& DMSO \\
Dodecanetiol capped Co nanoparticles & $n$-hexane, tollene \\
Pentatluorothiopenol capped Co nanoparticles & $\mathrm{MeOH}$. EtOH \\
Mercaptoundecanoic acid capped Co & water \\
nanoparticles & \\
\hline
\end{tabular}



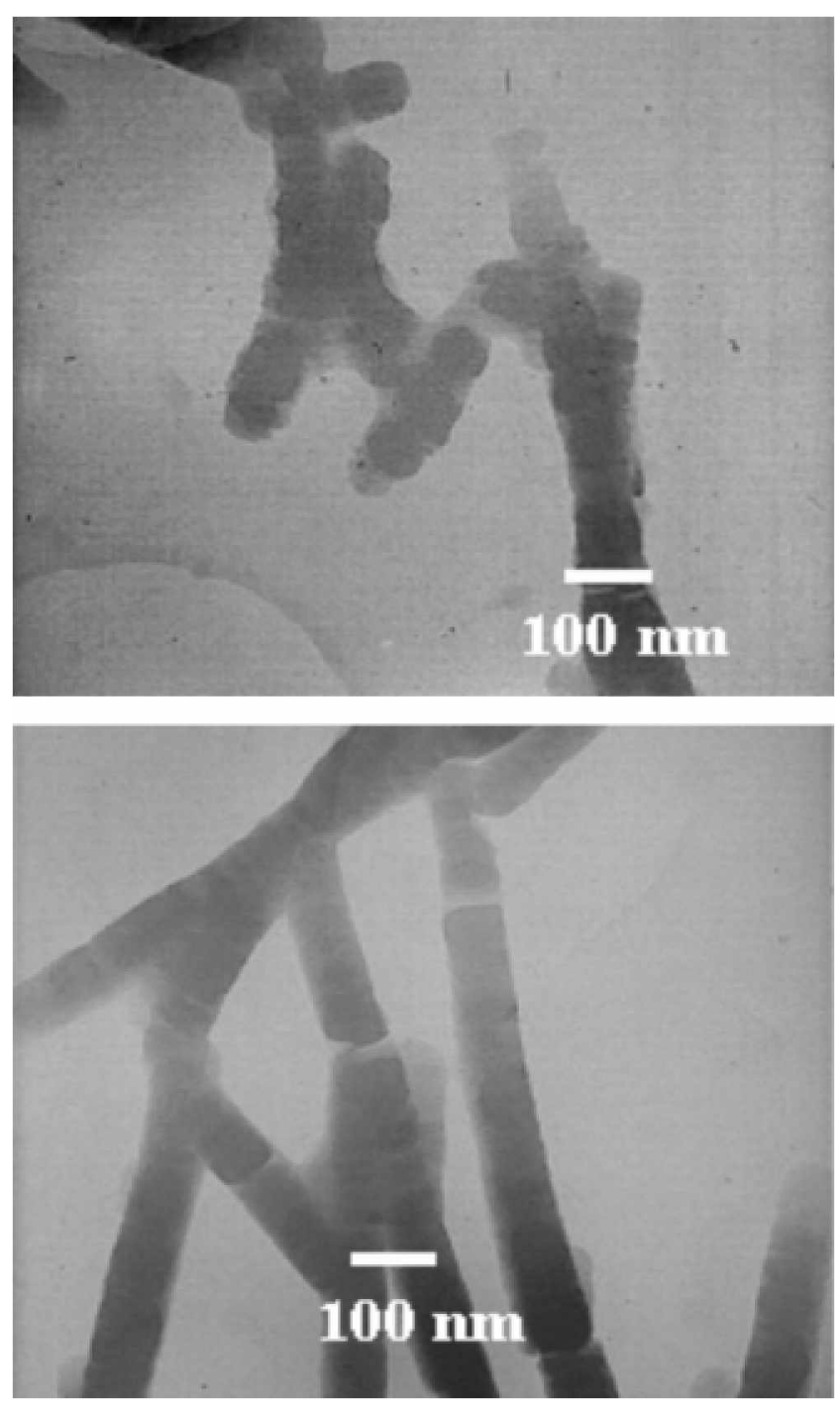

Figure 3. TEM pictures of magnetically aligned cobalt nanoparticles. Cobalt nanoparticles (synthetic condition: micelle concentration = 0.3 and water content $=40 \mu \mathrm{L}$ ) were magnetically aligned by slow oxidation with $\theta_{2}$ under the external magnetic field

$n$-hexane diethyl ether, and chloroform. However. dodecanethiol(DT)-capped Co nanoparticles are soluble in $n$-hexane and toluene. which are relatively nonpolar. Both TP- and DT-capped Co nanoparticles were insoluble in very polar solvents such as alcohols. while pentafluorothiophenol (PFTP)-capped Co nanoparticles show high solubility in alcohols. However. none of these capped Co nanoparticles were soluble in water. In order to prepare a water-soluble nanoparticle mercaptoundecanoic acid (MUA) was chosen as a capping ligand having ionic character and the resulting MUA-capped Co nanoparticles were very soluble only in water after the terminal group was changed to carboxylate salt by adjusting the $\mathrm{pH}$.

Surface derivatization was also confirmed by checking the FT-IR spectra of these organic derivatized (capped) cobalt nanoparticles. For TP-capped Co nanoparticles. peaks corresponding to $\mathrm{C}=\mathrm{C}_{\text {(arom }, l}$ appeared at $1577,1474,1438,1082$. 1023. 738 and $687 \mathrm{~cm}^{-1}$ and the stretching peak from $\mathrm{C}-\mathrm{H}$

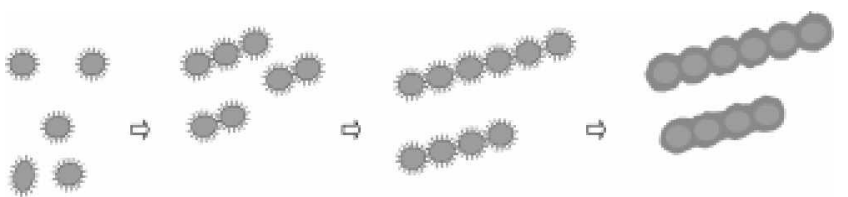

Scheme 1. The schematic diagram of the mechanism for formation of rod-like morphology.

appeared at $3065 \mathrm{~cm}^{-1}$. However, these peaks were slightly shifted lower frequencies compared with the neat organic capping materials due to the binding on the surface metal sites. For DT-capped Co nanoparticles. the detailed spectral features in the spectra of nanoparticles and neat dodecanethiol (not shown here) are also not so different but slightly shifted with respect to one another. $\mathrm{CH}_{2}$ symmetric and asymmetric stretching bands appear at 2853 and $2924 \mathrm{~cm}^{-1}$. respectively for neat dodecanethiol. but the corresponding bands are observed at slightly lower frequencies in the dodecanethiol-capped Co nanoparticles sample. at around 2830 and $2910 \mathrm{~cm}^{-1}$

Although these surface derivatized Co nanoparticles are stable against the formation of aggregates and no oxidation reaction proceeds at a detectable rate under inert nitrogen atmosphere. they tend to be slowly oxidized under ambient air to generate cobalt oxide causing insoluble aggregated precipitates. We have accidentally found a very interesting nanofabrication process which forms oxide-coated Co nanorods by slow oxidation of Co nanoparticles under an extemal magnetic field as shown in Figure 3. These TEM inages were taken after different collecting periods with an extemal magnetic field. After Co nanoparticles of about $\mathbf{5 0}$ $\mathrm{nm}$ in size. which were obtained in the freshly prepared solution. as shown in Figure 1(b). were kept in a magnetic field for a week. we observed that some of the nanoparticles were successively comnected with each other to give nanorods having a length of several microns (Fig. 3). Although the exact mechanism for the formation of this structure is not y'et clear. we speculate that it may be a result of alignment caused by an extemal magnetic field. A schematic diagran of the mechanism proposed is depicted in Scheme 1. Although capping materials are certainly present on Co nanoparticles. their influence on the particle is probably weak, allowing the dynamic attaching/detaching equilibrium. The reason is that samples are not used in conjunction with absolute methanol during the precipitation and are not stored under nitrogen during the application of the magnetic field. With time, the surface of thiophenol capped Co nanoparticles may be chemisorbed with dissolved oxygen. This leads to the formation of the thin oxide layer. The oxide layer wraps around the adjacent $C o$ particles, leading to the fomation of a peanut-shaped $\mathrm{Co}$ nanodimer and the nanooligomer. and ultimately a nanorod. A similar nanorod structure formation mechanism has been reported during the reduction of $\mathrm{b}-\mathrm{FeOOH}$ nanoparticle to metallic $\mathrm{Fe}$. even though there was no long range ordering due to the lack of an external field effect. ${ }^{3+}$

This hypothesis could be supported by the results of XPS 
investigation on these nanorods. The Co 2p XPS spectrum of nanorods. generated from thiophenol-capped Co nanoparticles without Ar ion sputtering (i.e., the outmost surface of nanorods). showed the Co $2 \mathrm{p}_{3}$ and $C_{0} 2 \mathrm{p}_{1: \text { peaks }}$ at the binding energy of 782.9 and $795.7 \mathrm{eV}$. confirming the presence of $\mathrm{Co}_{3} \mathrm{O}_{4}$ on the nanorod surfaces. which is the most stable oxide at room temperature. ${ }^{35}$ From the XPS spectrum of nanorods after Ar ion sputtering for 1 min. Co $2 \mathrm{p}$ signals shift to a lower binding energy of $778.2 \mathrm{eV}$ (Co $\left.2 \mathrm{p}_{30}\right)$ and $793.3 \mathrm{eV}\left(\mathrm{Co} 2 \mathrm{p}_{10}\right)$, in good agreement with the literature values for pure Co ${ }^{35}$ confirming the surface oxide layer was removed and the core Co metal was exposed

\section{Conclusion}

In this paper. we demonstrated that cobalt nanoparticles could be synthesized in a controlled manner by employing a reverse micelle technique. The sizes of Co nanoparticles increased from $8 \mathrm{~nm}$ to $50 \mathrm{~nm}$ as the water content was increased. Moreover. the shape of the nanoparticles could be adjusted from spherical to needle-shaped by increasing the micelle concentration. Furthemore, well-defined $\mathrm{Co}_{3} \mathrm{O}_{4}$ nanorods could be fabricated from Co nanoparticles by an external magnetic field. The surface capped Co nanoparticles with various organic ligands showed controllable solubility characteristics as expected: thiophenol (TP)-capped Co nanoparticles are soluble in relatively polar solvents such as pyridine. MIBK, and DMSO: dodecanethiol (DT)-capped Co nanoparticles are soluble in relatively nonpolar solvents such as $n$-hexane and toluene: pentafluorothio-phenol (PFTP)-capped Co nanoparticles are soluble in very polar alcohols such as methanol and ethanol: and 11-mercaptoundecanoic acid (MUA)-capped Co nanoparticles are very soluble in water.

Acknowledgment. The financial support from the Interdisciplinary Research Progran (Grant No. 1999-2-121-0045) of the KOSEF is greatly achnowledged. S. M. Choi is grateful for the award of a BK21 fellowship.

\section{References}

1. Shi. Y: Sarito. K.: Ishikura. H.: Hiramoto. T. J. Appl. Phns. 1998. 84. 2358.

2. Tiwar, S.: Rana. F.: Hanafi H. Hartstein. E.; Crabbe, F. Chan. K. Appl. P1s. Lett. 1996, 68.1377.
3. Welser, J. J.: Tiwar, S.; Riston, S.; Lee, K. Y.: Lee. Y. IEEE Electron Device Lett. 1997. 18. 278.

4. Murray. C. B.: Kagan1. C. R.: Bawendi. M. G. Science 1995. 270. 1335.

5. Carap. S. H.: Lu, P.-L.; He. Y. IEEE Trance Magn. 1997. 33. 978.

6. Raj. K.: Moskowitz, R. J. Magnt. Magnt. Matet 1990. 85, 233.

7. Rieke, R. D. Crit. Rev: Sitrf. Chent 1999, 1, 131.

8. Petit. C.: Pilen. M. P. J. Magn Magn. Mater 1997.166. 82.

9. Chen. T. P.: Sorensen. C. M.: Klabunde. K. J. Plys. Rev B 1995. 51. 11527.

10. Nagy. T. B.: Barette. D.: Fonseca. A.: Teunieau. L.: Monnoyer. P.: Piedigrosso, P.: Ravert. I; Verfaillie. J. P.: Wathelet, A. NTO ASI Ser. Ser. $31996,18,71$

11. Suslick. K. S.: Fang. M.: Hyeon, R.: Cichowlas, A. A. Mater: Res. Soc. Simp. Proc. 1994. 351. 443.

12. Yin. T. S.: Wang. Z. L. Nonostrict Mater 1999. 11.845

13. Apsel. S. E.: Emmert. J. W.: Deng. J.: Bloomfield. L. A. Phys. Rev: Lett. 1996, 76. 1441

14. Bucher, J. P.: Douglass, D. C.; Deng. J.; Bloofield, L. A. Phws. Rev: Lett. 1991, 66.3052.

15. Isabelle. M. L.: Billas. T. A.: Berker. T. A.: Chatelain. A.: DeHeer. W. A. Phus. Rev Lett 1993. 71. 4067.

16. Murray. C. B.: Norris. D. J.: Bawendi. M. G. J. Am. Chem Soc. 1993. 115,8706 .

17. Motte. L.: Pileni. M. P. Applied Surface Science 2000, 16t.60.

18. Prozorov. T; Kataby, G.'; Prozorov, R; Gedanken. A. Thin Solid Films 1999. 340. 189

19. Sun. S.: Murray. C. B. J. Appl. Phus. 1999. 85.4325

20. Lubbe. A. S.: Bergemann. C.: Brock. F.: McClure. D. G. J. Magn Magn. Mater: 1999. 194, 149

21. Popplewell. J.; Sakihnini. L. J. Magn. Magn. Mater 1995. 1t9. 72

22. Lin. X. M.: Sorensen. C. M.: Klabunde. K. T.: Hadjipanayis. G. C Langmiir 1998. 14.7140.

23. Ammarego. W. L. F.: Perrin. D. D. Purification of Laboraty Chemicals, th Ed.: Butterworth: Heinemann, 1996.

24. Yin. J. S.: Wang. Z. L. Namostmt. Mater 1999, M. 845 .

25. Pileni. M. P. Supramolectlar Science 1998, 100.3.

26. Kumer. S.: Aswal. V. K.: Singh. H. N.: Goyal. P. S.: Kabir-ud-Din Langnuir 1994. 10. 4069.

27. Zhao. G. X.: Li. X. G. J. Colloid Interface Sci. 1991. IH4. 185.

28. Durga Prasad. Ch.: Singh. H. N.: Goval. P. S.; Srinivasa Rao. K. L. J. Colloidhterface Sci, 1993, 155, 415.

29. Reekmans, S. Luo, H; wan der Auweraer, M; de Schryver. F. C. Langnuir 1990. 6.628.

30. Puntes. V. F.: Krishtnant. K. M.: Alivisatos. A. P. Science 2001. 291.2115

31. Lisiecki. I.: Billoudet. F, Pileni. M. P. J. Phws. Chent. 1996, 100. 4160.

32. Petit. C.; Taleb. A.: Pileni. M. P. J. Plns. Chem. B 1999, 103. 1805.

33. Tombolom. M.: Herikssen. U. d. Phys. Chem. B 1997. 101. 6028.

34. Chen1. M.: Nikles. D. E. J. Appl. Phys. 1999. 85.5504.

35. Oku, M.; Sato. Y. Appl. Suff Sci. 1992. 55, 37 